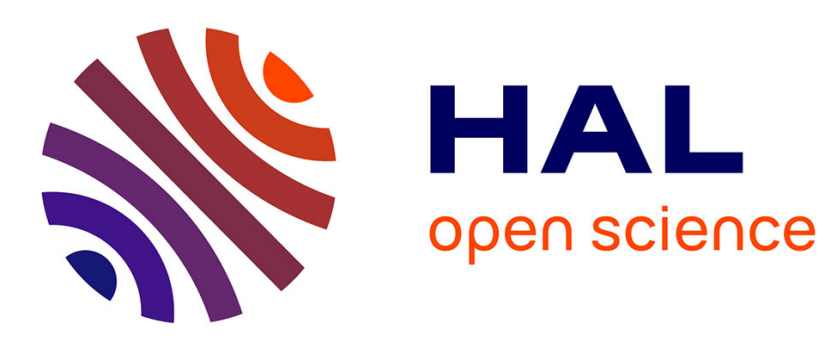

\title{
Iterative distributed outlier detection for wireless sensor networks: Equilibrium and convergence analysis
}

Wenjie Li, Francesca Bassi, Davide Dardari, Michel Kieffer, Gianni Pasolini

\section{To cite this version:}

Wenjie Li, Francesca Bassi, Davide Dardari, Michel Kieffer, Gianni Pasolini. Iterative distributed outlier detection for wireless sensor networks: Equilibrium and convergence analysis. 54th IEEE Conference on Decision and Control (CDC), Dec 2015, Osaka, Japan. 10.1109/cdc.2015.7402677 . hal-01327824

\section{HAL Id: hal-01327824 \\ https://hal.science/hal-01327824}

Submitted on 7 Jun 2016

HAL is a multi-disciplinary open access archive for the deposit and dissemination of scientific research documents, whether they are published or not. The documents may come from teaching and research institutions in France or abroad, or from public or private research centers.
L'archive ouverte pluridisciplinaire HAL, est destinée au dépôt et à la diffusion de documents scientifiques de niveau recherche, publiés ou non, émanant des établissements d'enseignement et de recherche français ou étrangers, des laboratoires publics ou privés. 


\title{
Iterative Distributed Outlier Detection for Wireless Sensor Networks: Equilibrium and Convergence Analysis
}

\author{
Wenjie Li $^{1}$, Francesca Bassi ${ }^{1,2}$, Davide Dardari ${ }^{3}$, Michel Kieffer ${ }^{1,4}$, and Gianni Pasolini ${ }^{3}$
}

\begin{abstract}
This paper analyzes a distributed outlier detection mechanism for wireless sensor networks. Outliers are data produced by sensors which are corrupted in a way that cannot simply be explained by the effects of the measurement noise. The outlier detection mechanism assumes some generic test to be available, which is only able to determine whether a set of data contains outliers, without being able to determine which data are outliers. The focus of this paper is on the equilibrium and stability analysis of the proposed iterative distributed outlier detection mechanism. Some sufficient conditions to be satisfied by the generic outlier detection test are established. Simulation results are then provided for an outlier model.
\end{abstract}

\section{INTRODUCTION}

Outliers are data produced by sensors and corrupted in a way that cannot be explained simply by the effects of the measurement noise. In classical centralized estimation, many efforts have been made to design estimators robust to outliers, see [1] and the references therein. In a distributed estimation setting, e.g., via a Wireless Sensor Network (WSN), these techniques are more difficult to put at work, due to communication constraints, which prevent the availability of a large amount of data at the sensing nodes of the WSN.

The aim of this paper is to present a variant of the iterative distributed outlier detection mechanism for WSN recently proposed in [2] and to analyze its equilibrium and convergence properties. This detection mechanism assumes the availability of some generic test, only able to determine whether a set of data coming from various sensors contains outliers, without being able to determine which data are outliers. This assumption is realistic, e.g., when a sensing node has to decide whether it produces outliers considering only the few data it has collected from its neighbors. The main difference with [2] is in the decision rule of the iterative mechanism, which improves its performance and facilitates its analysis. Sufficient conditions to be satisfied by the generic outlier detection test to ensure the existence of the equilibrium and its local asymptotic stability are established.

The paper is organized as follows. Section II provides some related work. Section III recalls some notations and describes the system model. Section IV recalls the distributed

\footnotetext{
*This work has been partly supported by the NEWCOM\# NoE

${ }^{1}$ W. Li, F. Bassi, and M. Kieffer are with Laboratoire des Signaux et Systèmes (L2S, UMR CNRS 8506) CNRS-CentraleSupelecUniversité Paris-Sud, 3, rue Joliot Curie 91192 Gif-sur-Yvette, France firstname. namedlss.supelec.fr

${ }^{2}$ F. Bassi is also with ESME-Sudria, 94200 Ivry-sur-Seine, France

${ }^{3}$ D. Dardari and G. Pasolini are with CNIT, DEI, University of Bologna, Italy. firstname.name@unibo.it

${ }^{4}$ M. Kieffer is also with LTCI Telecom ParisTech, 75013 Paris, France and Institut Universitaire de France, 75005 Paris, France
}

outlier detection mechanism and describes the sufficient conditions the generic test has to satisfy for uniqueness and local asymptotic stability of the outlier detection mechanism. Proofs of all results are given in Section V. Finally, some simulation examples are considered in Section VI-A before concluding the paper in Section VII.

\section{RELATED WORK}

Many distributed fault detection (DFD) algorithms have already been proposed in the literature. In [3] a purely localized algorithm is proposed to identify faulty sensors. The local test is based on the comparison between the local measurement and the median of the measurements of the neighbors. The algorithm devised in [4] allows each node to estimate its own functioning status by using a modified majority voting. Then the status of neighbors are collected and associated to their measurements to obtain a more reliable estimation. Other DFD algorithms can be found in [5], [6], [7], [8], where different detection strategies were presented. For example, [5], [6] proposed weighted-median and weighted average criteria, respectively. Intermittent faults in sensing and in communication are considered in [7], which proposes an adaptive DFD algorithm with a decision threshold adjusted at each round.

Instead of focusing on the strategy of local outlier detection test, the DFD algorithm proposed in [2] aims at performing the fault detection in an economic way by reducing the complexity of the decision rule and reducing the communication overhead. The generic local outlier detection test in this work has only to detect the presence of outliers in a set of data. This setting is similar to that considered in group testing (GT) [9], [10], which aims at identifying a small number of defective items within a large population, with a small amount of tests.

\section{Notations And System Model}

\section{A. Network model}

Consider a set of sensor nodes $\mathcal{S}$ deployed uniformly and independently over an infinite plane, with spatial density $\rho$. Assume that an arbitrary node $i$ can only communicate with the nodes in its neighborhood $\mathcal{N}_{i}=\left\{j \in \mathcal{S} \mid 0<r_{i, j} \leq R_{0}\right\}$, where $r_{i, j}$ is the distance between nodes $i$ and $j$, and $R_{0}$ is the communication range. Let $\mathcal{U}_{i}=\mathcal{N}_{i} \cup\{i\}$. The number of elements $\left|\mathcal{N}_{i}\right|$ of $\mathcal{N}_{i}$ follows a Poisson distribution

$$
\mathbb{P}\left\{\left|\mathcal{N}_{i}\right|=n\right\}=\frac{\mu^{n}}{n !} \exp (-\mu),
$$

with average $\mu=\rho \pi R_{0}^{2}$, see [11]. 
Define $\mathcal{D} \subseteq \mathcal{S}$ as the set of nodes with defective sensors, which produce outliers, i.e., data whose characteristics differ significantly from those produced by non-defective (good) sensors. Similarly, define $\mathcal{G}=\mathcal{S} \backslash \mathcal{D}$ as the set of nodes with good sensors. Here, we assume that only the sensing capabilities of nodes may be defective. Altered communication and computation capabilities are not considered. Let $\theta_{i} \in\{0,1\}$ denote the status of the sensor of an arbitrary node $i \in \mathcal{S}$. One has $\theta_{i}=1$ if $i \in \mathcal{D}$ and $\theta_{i}=0$ if $i \in \mathcal{G}$. Moreover, $\theta_{i}$ and $\theta_{j}$ are assumed to be independent for any $i, j \in \mathcal{S}$ with $i \neq j$. As a consequence,

$$
\mathbb{P}\left\{\left|\mathcal{N}_{i} \cap \mathcal{G}\right|=n_{\mathrm{g}},\left|\mathcal{N}_{i} \cap \mathcal{D}\right|=n_{\mathrm{d}}\right\}=\frac{\mu_{\mathrm{d}}^{n_{\mathrm{d}}} \mu_{\mathrm{g}}^{n_{\mathrm{g}}}}{n_{\mathrm{d}} ! n_{\mathrm{g}} !} \exp (-\mu),
$$

where $\mu_{\mathrm{d}}$ and $\mu_{\mathrm{g}}$ denote the average values of $\left|\mathcal{N}_{i} \cap \mathcal{D}\right|$ and $\left|\mathcal{N}_{i} \cap \mathcal{G}\right|$, respectively.

\section{B. Local outlier detection test}

At a given time instant, let the random vector $M_{i} \in$ $\mathbb{R}^{n}$ denote the data provided by node $i$. Consider $\mathbf{M}_{\mathcal{A}}=$ $\left[M_{i}\right]_{i \in \mathcal{A}} \in \mathbb{R}^{n|\mathcal{A}|}$, the random vector of data provided by a set of nodes $\mathcal{A} \subseteq \mathcal{S}$. For a given realization $\mathbf{m}_{\mathcal{A}} \in \mathbb{R}^{n|\mathcal{A}|}$ of $\mathbf{M}_{\mathcal{A}}$, denote $T\left(\mathbf{m}_{\mathcal{A}}\right)$ the outcome of some local outlier detection test (LODT)

$$
T\left(\mathbf{m}_{\mathcal{A}}\right)= \begin{cases}0, & \text { if no outlier is detected in } \mathbf{m}_{\mathcal{A}}, \\ 1, & \text { otherwise }\end{cases}
$$

Even if $\mathcal{A} \cap \mathcal{D} \neq \emptyset$, the noise characteristics of defective sensors may produce some realizations $\mathbf{m}_{\mathcal{A}}$ that do not allow the detection of the outliers. Thus, one introduces the probability of outlier detection

$$
q_{\mathrm{D}}\left(\mathbf{M}_{\mathcal{A}}\right)=\mathbb{P}\left\{T\left(\mathbf{M}_{\mathcal{A}}\right)=1 \mid \mathcal{A} \cap \mathcal{D} \neq \emptyset\right\},
$$

and the probability of false alarm

$$
q_{\mathrm{FA}}\left(\mathbf{M}_{\mathcal{A}}\right)=\mathbb{P}\left\{T\left(\mathbf{M}_{\mathcal{A}}\right)=1 \mid \mathcal{A} \cap \mathcal{D}=\emptyset\right\}
$$

In this work we consider only tests satisfying the following properties.

Property 1: Let $n_{\mathrm{g}}=|\mathcal{A} \cap \mathcal{G}|$ and $n_{\mathrm{d}}=|\mathcal{A} \cap \mathcal{D}|$, then

$q_{\mathrm{FA}}\left(\mathbf{M}_{\mathcal{A}}\right)=q_{\mathrm{FA}}\left(n_{\mathrm{g}}\right), \quad q_{\mathrm{D}}\left(\mathbf{M}_{\mathcal{A}}\right)=q_{\mathrm{D}}\left(n_{\mathrm{g}}, n_{\mathrm{d}}\right)$.

Property 2: Let $k$ be an arbitrary node in $\mathcal{A}$, then

$$
\left\{\begin{array}{l}
\mathbb{P}\left\{T\left(\mathbf{M}_{\mathcal{A} \backslash\{k\}}\right)=1 \mid T\left(\mathbf{M}_{\mathcal{A}}\right)=0, \mathcal{A} \cap \mathcal{D} \neq \emptyset\right\}=0, \\
\mathbb{P}\left\{T\left(\mathbf{M}_{\mathcal{A} \backslash\{k\}}\right)=1 \mid T\left(\mathbf{M}_{\mathcal{A}}\right)=0, \mathcal{A} \cap \mathcal{D}=\emptyset\right\}=0 .
\end{array}\right.
$$

In Property 1, we consider only the class of LODTs $T$ acting on the data vector $\mathbf{M}_{\mathcal{A}}$ in a way that disregards the knowledge of the identity of the node producing the data. For this reason, (4) and (5) depend only on the number and on the status (good or defective) of the sensors in $\mathcal{A}$. Moreover, we focus on tests allowing an easier identification of an outlier as $|\mathcal{A}|$ increases. Property 2 implies that if an outlier is not discovered testing the whole vector of data $\mathbf{m}_{\mathcal{A}}$, then it cannot be discovered testing any sub-vector of $\mathbf{m}_{\mathcal{A}}$.

\section{Problem Statement and Main Results}

\section{A. Iterative Distributed Outlier Detection Algorithm}

This section presents a variant of the iterative DFD algorithm proposed in [2]. The aim of Algorithm 1 is to detect nodes with defective sensors in the WSN and to reduce their impact, while keeping the probability of false alarm small. At each round $\ell$ of the iterative algorithm, each node $i$ has to provide an estimate $\widehat{\theta}_{i}^{(\ell)}$ of the status of its sensor. Then, the estimates $\widehat{\theta}_{i}^{(\ell)}$ will affect the set of data considered by node $i$ at round $\ell+1$, i.e., $\widehat{\mathcal{U}}_{i}^{(\ell+1)}=\left\{j \in \mathcal{U}_{i}\right.$ s.t. $\left.\widehat{\theta}_{j}^{(\ell)}=0\right\}$. Estimates may thus be updated at the next round.

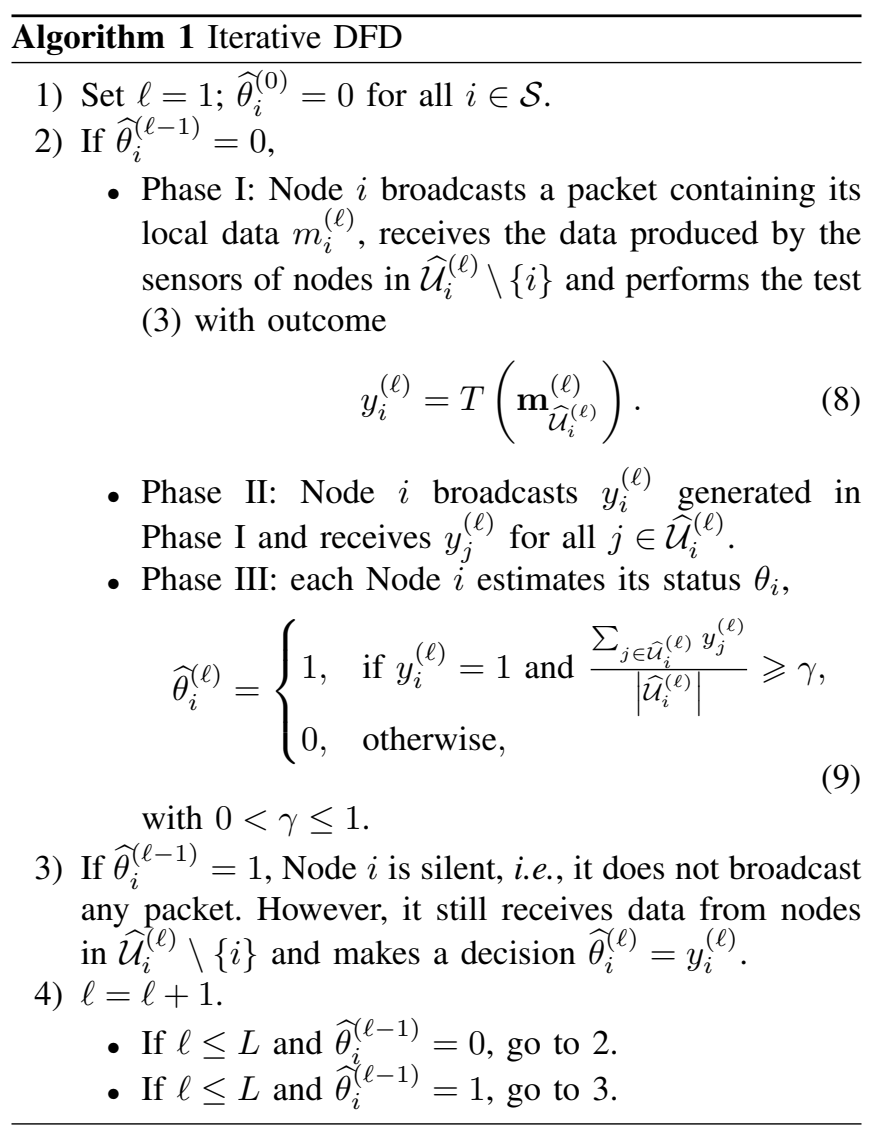

At iteration $\ell=1$, each node performs the operations described in Step 2. After data collection from its own sensor and the sensors of neighboring nodes, each node $i$ locally decides whether there exists an outlier in $\mathbf{m}_{\widehat{\mathcal{U}}_{i}^{(\ell)}}^{(\ell)}$ and broadcasts its binary decision $y_{i}^{(\ell)}$ to its neighbors. At the end of Step $2, \widehat{\theta}_{i}^{(\ell)}$ is obtained by applying (9). One has $\widehat{\theta}_{i}^{(\ell)}=1$ if node $i$ detects an outlier and if the proportion of neighboring nodes detecting an outlier in their collected data is larger than $\gamma$. Note that $y_{i}^{(\ell)}$ depends only on the nodes in $\widehat{\mathcal{U}}_{i}^{(\ell)}$ and is not affected by the presence of outliers outside.

When $\mu_{\mathrm{d}} \geqslant 1$, a large amount of non-defective sensors may be diagnosed as defective. In Step 3 , if $\widehat{\theta}_{i}^{(\ell-1)}=1$, node $i$ becomes silent and stops broadcasting its measurements in round $\ell$. However, node $i$ still performs the LODT: this gives it a chance to become active again at iteration $\ell+1$ when it 
erroneously considered its sensor as defective at iteration $\ell$.

\section{B. Analysis of the DFD algorithm}

Let the pair $\left(\theta_{i}, \hat{\theta}_{i}^{(\ell)}\right)$ denote the state of Node $i$. Among the four possible states, $(0,0)$ and $(1,1)$ are states resulting from a correct decision, $(0,1)$ corresponds to a false alarm, and $(1,0)$ corresponds to a non-detection. Let $\mu_{\theta_{i}, \hat{\theta}_{i}^{(\ell)}}^{(\ell)}$ denote the density of sensors in the state $\left(\theta_{i}, \hat{\theta}_{i}^{(\ell)}\right)$. The aim of what follows is to characterize the evolution of $\mu_{\theta_{i}, \hat{\theta}_{i}}^{(\ell)}$ to determine whether Algorithm 1 converges to a unique equilibrium state.

Before the first round of the adaptive algorithm, one has $\mu_{00}^{(0)}=\mu_{\mathrm{g}}, \mu_{10}^{(0)}=\mu_{\mathrm{d}}$, and $\mu_{01}^{(0)}=\mu_{11}^{(0)}=0$. Define

$$
\left\{\begin{array}{l}
f_{1}\left(\mu_{\mathrm{g}}, \mu_{\mathrm{d}}\right)=\mathbb{P}\left\{Y_{i}^{(1)}=1 \mid \theta_{i}=0\right. \\
f_{2}\left(\mu_{\mathrm{g}}, \mu_{\mathrm{d}}\right)=\mathbb{P}\left\{Y_{i}^{(1)}=1 \mid \theta_{i}=1\right.
\end{array}\right\}
$$

as the probability of having $Y_{i}^{(1)}=1$. Note that $f_{1}$ and $f_{2}$ are functions of $\mu_{\mathrm{g}}$ and $\mu_{\mathrm{d}}$ due to Property 1 . Similarly, define

$$
\left\{\begin{array}{l}
P_{\mathrm{FA}}\left(\mu_{\mathrm{g}}, \mu_{\mathrm{d}}\right)=\mathbb{P}\left\{\hat{\theta}_{i}^{(1)}=1 \mid \theta_{i}=0\right\} \\
P_{\mathrm{D}}\left(\mu_{\mathrm{g}}, \mu_{\mathrm{d}}\right)=\mathbb{P}\left\{\hat{\theta}_{i}^{(1)}=1 \mid \theta_{i}=1\right\}
\end{array}\right.
$$

as the probability of having $\hat{\theta}_{i}^{(1)}=1$ in the first iteration, at the end of Step 2. The properties of $f_{1}, f_{2}, P_{\mathrm{FA}}$, and $P_{\mathrm{D}}$ are discussed in Section $\mathrm{V}-\mathrm{A}$.

At any iteration $\ell, \mu_{00}^{(\ell)}+\mu_{01}^{(\ell)}=\mu_{\mathrm{g}}$ and $\mu_{10}^{(\ell)}+\mu_{11}^{(\ell)}=\mu_{\mathrm{d}}$. Since the actual status of a sensor is assumed constant during the iterations of the DFD algorithm, the only possible transitions are between states $(0,0)$ and $(0,1)$ and between states $(1,0)$ and $(1,1)$. The evolution of the densities between iterations $\ell$ and $\ell+1$ is given by

$$
\left[\begin{array}{c}
\mu_{00}^{(\ell+1)} \\
\mu_{01}^{(\ell+1)} \\
\mu_{10}^{(\ell+1)} \\
\mu_{11}^{(\ell+1)}
\end{array}\right]=\left[\begin{array}{cccc}
P_{0,00}^{(\ell)} & P_{0,10}^{(\ell)} & 0 & 0 \\
P_{0,01}^{(\ell)} & P_{0,11}^{(\ell)} & 0 & 0 \\
0 & 0 & P_{1,00}^{(\ell)} & P_{1,10}^{(\ell)} \\
0 & 0 & P_{1,01}^{(\ell)} & P_{1,11}^{(\ell)}
\end{array}\right]\left[\begin{array}{c}
\mu_{00}^{(\ell)} \\
\mu_{01}^{(\ell)} \\
\mu_{10}^{(\ell)} \\
\mu_{11}^{(\ell)}
\end{array}\right],
$$

with $P_{a, b c}^{(\ell)}=\mathbb{P}\left\{\hat{\theta}_{i}^{(\ell+1)}=c \mid \hat{\theta}_{i}^{(\ell)}=b, \theta_{i}=a\right\}$, for any $a, b, c \in\{0,1\}$. The sensors considered as defective in the previous iteration are silent, so all the transition probabilities are functions of $\mu_{00}^{(\ell)}$ and $\mu_{10}^{(\ell)}$. When $\hat{\theta}_{i}^{(\ell-1)}=0$, Step 2 is performed and

$$
\begin{cases}P_{0,01}^{(\ell)}=P_{\mathrm{FA}}\left(\mu_{00}^{(\ell)}, \mu_{10}^{(\ell)}\right), & P_{0,00}^{(\ell)}=1-P_{0,01}^{(\ell)}, \\ P_{1,01}^{(\ell)}=P_{\mathrm{D}}\left(\mu_{00}^{(\ell)}, \mu_{10}^{(\ell)}\right), & P_{1,00}^{(\ell)}=1-P_{1,01}^{(\ell)} .\end{cases}
$$

When $\hat{\theta}_{i}^{(\ell-1)}=1$, Step 3 yields $\widehat{\theta}_{i}^{(\ell)}=y_{i}^{(\ell)}$ and

$$
\begin{cases}P_{0,11}^{(\ell)}=f_{1}\left(\mu_{00}^{(\ell)}, \mu_{10}^{(\ell)}\right), & P_{0,10}^{(\ell)}=1-P_{0,11}^{(\ell)}, \\ P_{1,11}^{(\ell)}=f_{2}\left(\mu_{00}^{(\ell)}, \mu_{10}^{(\ell)}\right), & P_{1,10}^{(\ell)}=1-P_{1,11}^{(\ell)} .\end{cases}
$$

One may rewrite (12) as follows

$$
\left\{\begin{array}{l}
\mu_{00}^{(\ell+1)}=F_{1}\left(\mu_{00}^{(\ell)}, \mu_{10}^{(\ell)}\right) \\
\mu_{10}^{(\ell+1)}=F_{2}\left(\mu_{00}^{(\ell)}, \mu_{10}^{(\ell)}\right)
\end{array}\right.
$$

with

$$
\left\{\begin{array}{l}
F_{1}\left(\mu_{00}^{(\ell)}, \mu_{10}^{(\ell)}\right)=\mu_{00}^{(\ell)}\left(f_{1}-P_{\mathrm{FA}}\right)+\mu_{\mathrm{g}}\left(1-f_{1}\right), \\
F_{2}\left(\mu_{00}^{(\ell)}, \mu_{10}^{(\ell)}\right)=\mu_{10}^{(\ell)}\left(f_{2}-P_{\mathrm{D}}\right)+\mu_{\mathrm{d}}\left(1-f_{2}\right) .
\end{array}\right.
$$

To lighten notations, the arguments $\mu_{00}^{(\ell)}$ and $\mu_{10}^{(\ell)}$ of $P_{\mathrm{FA}}, P_{\mathrm{D}}$, $f_{1}$, and $f_{2}$ have been omitted in (16), and in what follows when there is no confusion. Note that (15) describes a nonlinear system, as all the transition probabilities depend on the densities $\mu_{00}^{(\ell)}$ and $\mu_{10}^{(\ell)}$.

\section{Equilibrium analysis}

Let $\mu_{00}^{*}$ and $\mu_{10}^{*}$ be the values at equilibrium of $\mu_{00}^{(\ell)}$ and $\mu_{10}^{(\ell)}$, respectively. From (15), one deduces that $\mu_{00}^{*}$ and $\mu_{10}^{*}$ should satisfy

$$
\begin{aligned}
\frac{\mu_{00}^{*} P_{\mathrm{FA}}\left(\mu_{00}^{*}, \mu_{10}^{*}\right)}{\mu_{\mathrm{g}}-\mu_{00}^{*}} & =1-f_{1}\left(\mu_{00}^{*}, \mu_{10}^{*}\right), \\
\frac{\mu_{10}^{*} P_{\mathrm{D}}\left(\mu_{00}^{*}, \mu_{10}^{*}\right)}{\mu_{\mathrm{d}}-\mu_{10}^{*}} & =1-f_{2}\left(\mu_{00}^{*}, \mu_{10}^{*}\right) .
\end{aligned}
$$

In what follows, one determines the properties (existence, unicity, and local stability) of the equilibrium state, which depend on the properties of the transition probabilities. First, one states sufficient conditions on the LODT, to get a unique equilibrium state in (15).

Proposition 1: Assume that the considered LODT satisfies Properties 1 and 2, and is such that

$$
\begin{gathered}
\mathbb{P}\left\{Y_{k}=1 \mid Y_{i}=1, \theta_{k}=\theta_{i}=0, k \in \mathcal{N}_{i}\right\}>1-\frac{4}{\mu_{\mathrm{g}}}, \\
q_{\mathrm{D}}(0,2)>1-\frac{4}{\mu_{\mathrm{d}}},
\end{gathered}
$$

then (17-18) admit a solution, and the equilibrium of (15) exists.

If $\mu_{\mathrm{g}}<4$ and $\mu_{\mathrm{d}}<4$, one has $1-4 / \mu_{\mathrm{g}}<0$ and $1-4 / \mu_{\mathrm{d}}<0$, then (19) and (20) hold for any kind of test satisfying Properties 1 and 2 . In fact, $\mu_{\mathrm{d}}$ has typically small values, thus (20) could be easily satisfied. In the situation where $\mu_{\mathrm{g}} \geqslant 4$, (19) constrains the test: considering two non-defective neighboring nodes $i$ and $k$, the conditional probability $\mathbb{P}\left\{Y_{k}=1 \mid Y_{j}=1\right\}$ should be larger than $1-4 / \mu_{\mathrm{g}}$. Notice that $Y_{i}$ and $Y_{k}$ are dependent, thus $\mathbb{P}\left\{Y_{k}=1 \mid Y_{j}=1\right\}$ is not small. However, if $\mu_{\mathrm{g}}$ goes large, (19) may be difficult to satisfy. The exact value of the lower bound depends on the type of LODT, see Section VI-A.

\section{Local asymptotic stability analysis}

Consider a linearization of the first equation of (15) around equilibrium with $\mu_{00}^{(\ell)}=\mu_{00}^{*}+\delta_{0}^{(\ell)}$ and $\mu_{10}^{(\ell)}=\mu_{10}^{*}+\delta_{1}^{(\ell)}$ around $\left(\mu_{00}^{*}, \mu_{10}^{*}\right)$, one gets

$$
\left(\begin{array}{l}
\delta_{0}^{(\ell+1)} \\
\delta_{1}^{(\ell+1)}
\end{array}\right)=\left[\begin{array}{ll}
A_{11} & A_{12} \\
A_{21} & A_{22}
\end{array}\right]\left(\begin{array}{l}
\delta_{0}^{(\ell)} \\
\delta_{1}^{(\ell)}
\end{array}\right)=\mathbf{A}\left(\begin{array}{c}
\delta_{0}^{(\ell)} \\
\delta_{1}^{(\ell)}
\end{array}\right),
$$


where

$$
\mathbf{A}=\left[\begin{array}{cc}
\frac{\partial F_{1}}{\partial \mu_{00}}\left(\mu_{00}^{*}, \mu_{10}^{*}\right) & \frac{\partial F_{1}}{\partial \mu_{10}}\left(\mu_{00}^{*}, \mu_{10}^{*}\right) \\
\frac{\partial F_{2}}{\partial \mu_{00}}\left(\mu_{00}^{*}, \mu_{10}^{*}\right) & \frac{\partial F_{2}}{\partial \mu_{10}}\left(\mu_{00}^{*}, \mu_{10}^{*}\right)
\end{array}\right] .
$$

The linearized system (21) is locally asymptotically stable if the eigenvalues of $\mathbf{A}$ are in the unit circle, which leads to the following proposition.

Proposition 2: Consider a LODT that satisfies Properties 1 and 2, as well as (19-20), and is such that

$$
\left\{\begin{array}{l}
A_{11} A_{22}-A_{12} A_{21}<1 \\
\left(1-A_{11}\right)\left(1-A_{22}\right)-A_{12} A_{21}>0 \\
\left(1+A_{11}\right)\left(1+A_{22}\right)-A_{12} A_{21}>0
\end{array}\right.
$$

then the equilibrium point $\left(\mu_{00}^{*}, \mu_{10}^{*}\right)$ is locally stable.

Providing generic stability conditions for all possible LODT is difficult. Nevertheless, for a given LODT, the local stability of (21) may be verified numerically. This requires an evaluation of the derivatives of $f_{1}, f_{2}, P_{\mathrm{FA}}$ and $P_{\mathrm{D}}$ with respect to $\mu_{00}$ and $\mu_{10}$ at equilibrium, see Section VI-A.

\section{Proofs}

In Section V-A we characterize analytically the transition probabilities $f_{1}, f_{2}, P_{\mathrm{FA}}$ and $P_{\mathrm{D}}$. Then the existence of the equilibrium (Proposition 1) is proved in Section V-B.

\section{A. Properties of transition probabilities}

One begins with the evaluation of the probability of having $Y_{i}=1$ for the LODT (3). This probability depends on the random variables $N_{\mathrm{g}}=\left|\mathcal{U}_{i} \cap \mathcal{G}\right|$ and $N_{\mathrm{d}}=\left|\mathcal{U}_{i} \cap \mathcal{D}\right|$. Define the function $h\left(n_{\mathrm{g}}, n_{\mathrm{d}}\right)$ as the probability of having $Y_{i}=1$ in a local test knowing that $N_{\mathrm{g}}=n_{\mathrm{g}}$ and $N_{\mathrm{d}}=n_{\mathrm{d}}$, i.e.,

$$
\begin{aligned}
h\left(n_{\mathrm{g}}, n_{\mathrm{d}}\right) & \stackrel{(a)}{=} \mathbb{P}\left\{Y_{i}=1 \mid N_{\mathrm{g}}=n_{\mathrm{g}}, N_{\mathrm{d}}=n_{\mathrm{d}}, \theta_{i}=0\right\} \\
& \stackrel{(b)}{=} \mathbb{P}\left\{Y_{i}=1 \mid N_{\mathrm{g}}=n_{\mathrm{g}}, N_{\mathrm{d}}=n_{\mathrm{d}}, \theta_{i}=1\right\} \\
& = \begin{cases}q_{\mathrm{FA}}\left(n_{\mathrm{g}}\right), & \text { if } n_{\mathrm{d}}=0, \\
q_{\mathrm{D}}\left(n_{\mathrm{g}}, n_{\mathrm{d}}\right), & \text { if } n_{\mathrm{d}} \neq 0 .\end{cases}
\end{aligned}
$$

Note that $(a)$ and $(b)$ hold as this probability does not depend on $\theta_{i}$, according to Property 1 .

Lemma 3: For any LODT $T$ satisfying Properties 1 and 2, one has

$$
\begin{aligned}
q_{\mathrm{FA}}\left(n_{\mathrm{g}}\right) & \leq q_{\mathrm{FA}}\left(n_{\mathrm{g}}+1\right), \\
q_{\mathrm{D}}\left(n_{\mathrm{g}}, n_{\mathrm{d}}\right) & \leq q_{\mathrm{D}}\left(n_{\mathrm{g}}+1, n_{\mathrm{d}}\right), \\
q_{\mathrm{D}}\left(n_{\mathrm{g}}, n_{\mathrm{d}}\right) & \leq q_{\mathrm{D}}\left(n_{\mathrm{g}}, n_{\mathrm{d}}+1\right) .
\end{aligned}
$$

Proof: First, note that both $q_{\mathrm{D}}$ and $q_{\mathrm{FA}}$ represent the probabilities of getting $Y=1$. One has, for any $k \in \mathcal{A}$

$$
\begin{aligned}
& \mathbb{P}\left\{T\left(\mathbf{M}_{\mathcal{A} \backslash\{k\}}\right)=1 \mid \mathcal{A} \cap \mathcal{D}=\emptyset\right\} \\
& \stackrel{(a)}{=} \mathbb{P}\left\{T\left(\mathbf{M}_{\mathcal{A} \backslash\{k\}}\right)=1, T\left(\mathbf{M}_{\mathcal{A}}\right)=1 \mid \mathcal{A} \cap \mathcal{D}=\emptyset\right\} \\
& \leq \mathbb{P}\left\{T\left(\mathbf{M}_{\mathcal{A}}\right)=1 \mid \mathcal{A} \cap \mathcal{D}=\emptyset\right\}
\end{aligned}
$$

where $(a)$ is obtained from (7). From (5) and (27), one gets

$$
q_{\mathrm{FA}}\left(\mathbf{M}_{\mathcal{A} \backslash\{k\}}\right) \leq q_{\mathrm{FA}}\left(\mathbf{M}_{\mathcal{A}}\right) .
$$

Suppose that $|\mathcal{A}|=n_{\mathrm{g}}+1$ with $n_{\mathrm{g}} \geq 1$, then based on Property 1 , (28) is equivalent to $q_{\mathrm{FA}}\left(n_{\mathrm{g}}\right) \leq q_{\mathrm{FA}}\left(n_{\mathrm{g}}+1\right)$, which proves (24). One may prove (25) and (26) in a similar way, by considering $\varphi(\mathcal{A})=1$ in the derivations.

Now consider $f_{1}$ and $f_{2}$ defined in (10):

$$
\begin{aligned}
& f_{1}\left(\mu_{\mathrm{g}}, \mu_{\mathrm{d}}\right)=\mathbb{P}\left\{Y_{i}=1 \mid \theta_{i}=0\right\} \\
& =\sum_{n_{\mathrm{g}}=1}^{\infty} \sum_{n_{\mathrm{d}}=0}^{\infty} \mathbb{P}\left\{Y_{i}=1 \mid N_{\mathrm{g}}=n_{\mathrm{g}}, N_{\mathrm{d}}=n_{\mathrm{d}}, \theta_{i}=0\right\} \\
& \cdot \mathbb{P}\left\{N_{\mathrm{g}}=n_{\mathrm{g}}, N_{\mathrm{d}}=n_{\mathrm{d}} \mid \theta_{i}=0\right\} \\
& =\sum_{n_{\mathrm{g}}=1}^{\infty} \sum_{n_{\mathrm{d}}=0}^{\infty} h\left(n_{\mathrm{g}}, n_{\mathrm{d}}\right) \frac{\mu_{\mathrm{d}}^{n_{\mathrm{d}}} \mu_{\mathrm{g}} n_{\mathrm{g}}-1}{n_{\mathrm{d}} !\left(n_{\mathrm{g}}-1\right) !} \exp \left(-\mu_{\mathrm{d}}-\mu_{\mathrm{g}}\right),
\end{aligned}
$$

$$
\begin{aligned}
& f_{2}\left(\mu_{\mathrm{g}}, \mu_{\mathrm{d}}\right)=\mathbb{P}\left\{Y_{i}=1 \mid \theta_{i}=1\right\} \\
& =\sum_{n_{\mathrm{g}}=0}^{\infty} \sum_{n_{\mathrm{d}}=1}^{\infty} h\left(n_{\mathrm{g}}, n_{\mathrm{d}}\right) \frac{\mu_{\mathrm{d}}^{n_{\mathrm{d}}-1} \mu_{\mathrm{g}}^{n_{\mathrm{g}}}}{\left(n_{\mathrm{d}}-1\right) ! n_{\mathrm{g}} !} \exp \left(-\mu_{\mathrm{d}}-\mu_{\mathrm{g}}\right) .
\end{aligned}
$$

Lemma 4: $f_{1}\left(\mu_{\mathrm{g}}, \mu_{\mathrm{d}}\right)$ and $f_{2}\left(\mu_{\mathrm{g}}, \mu_{\mathrm{d}}\right)$ are non-decreasing functions of $\mu_{\mathrm{g}}$ and $\mu_{\mathrm{d}}$.

Proof: One may easily show that

$$
\begin{array}{r}
\frac{\partial f_{1}}{\partial \mu_{\mathrm{g}}}=\sum_{n_{\mathrm{g}}=1}^{\infty} \sum_{n_{\mathrm{d}}=0}^{\infty} \frac{\mu_{\mathrm{d}}^{n_{\mathrm{d}}} \mu_{\mathrm{g}}^{n_{\mathrm{g}}-1}}{n_{\mathrm{d}} !\left(n_{\mathrm{g}}-1\right) !} \exp \left(-\mu_{\mathrm{d}}-\mu_{\mathrm{g}}\right) \\
\cdot\left(h\left(n_{\mathrm{g}}+1, n_{\mathrm{d}}\right)-h\left(n_{\mathrm{g}}, n_{\mathrm{d}}\right)\right) .
\end{array}
$$

From (31), one has $\frac{\partial f_{1}}{\partial \mu_{\mathrm{g}}} \geq 0$ as $h\left(n_{\mathrm{g}}, n_{\mathrm{d}}+1\right) \geq h\left(n_{\mathrm{g}}, n_{\mathrm{d}}\right)$ by Lemma 3. Similarly, one has $\frac{\partial f_{1}}{\partial \mu_{\mathrm{d}}} \geq 0, \frac{\partial f_{2}}{\partial \mu_{\mathrm{g}}} \geq 0$, and $\frac{\partial f_{2}}{\partial \mu_{\mathrm{d}}} \geq 0$.

Define now the conditional false alarm event

$\mathcal{E}_{i, \mathrm{FA}}^{n_{\mathrm{g}}, n_{\mathrm{d}}}=\left\{Y_{i}=1, \frac{\sum_{j \in \mathcal{U}_{i}} Y_{j}}{n_{\mathrm{g}}+n_{\mathrm{d}}} \geq \gamma \mid \theta_{i}=0, N_{\mathrm{g}}=n_{\mathrm{g}}, N_{\mathrm{d}}=n_{\mathrm{d}}\right\}$

representing, according to (9), the situation where the node $i$ with non-defective sensor diagnoses it as defective, knowing that $N_{\mathrm{g}}=n_{\mathrm{g}} \geq 1$ and $N_{\mathrm{d}}=n_{\mathrm{d}} \geq 0$. Let $\tau_{\mathrm{FA}}\left(n_{\mathrm{g}}, n_{\mathrm{d}}\right)=$ $\mathbb{P}\left\{\mathcal{E}_{i, \mathrm{FA}}^{n_{\mathrm{g}}, n_{\mathrm{d}}}\right\}$, then $P_{\mathrm{FA}}$ defined in (11) can be expressed as

$$
\begin{gathered}
P_{\mathrm{FA}}=\sum_{n_{\mathrm{g}}=1}^{\infty} \sum_{n_{\mathrm{d}}=0}^{\infty} \tau_{\mathrm{FA}}\left(n_{\mathrm{g}}, n_{\mathrm{d}}\right) \mathbb{P}\left\{N_{\mathrm{g}}=n_{\mathrm{g}}, N_{\mathrm{d}}=n_{\mathrm{d}} \mid \theta_{i}=0\right\} \\
=\sum_{n_{\mathrm{g}}=1}^{\infty} \sum_{n_{\mathrm{d}}=0}^{\infty} \tau_{\mathrm{FA}}\left(n_{\mathrm{g}}, n_{\mathrm{d}}\right) \frac{\mu_{\mathrm{d}}^{n_{\mathrm{d}}} \mu_{\mathrm{g}} n_{\mathrm{g}}-1 \exp \left(-\mu_{\mathrm{d}}-\mu_{\mathrm{g}}\right)}{n_{\mathrm{d}} !\left(n_{\mathrm{g}}-1\right) !}
\end{gathered}
$$

Similarly, consider a non-defective sensor $i$ : the conditional defective sensor detection event is

$\mathcal{E}_{i, \mathrm{D}}^{n_{\mathrm{g}}, n_{\mathrm{d}}}=\left\{Y_{i}=1, \frac{\sum_{j \in \mathcal{U}_{i}} Y_{j}}{n_{\mathrm{g}}+n_{\mathrm{d}}} \geq \gamma \mid \theta_{i}=1, N_{\mathrm{g}}=n_{\mathrm{g}}, N_{\mathrm{d}}=n_{\mathrm{d}}\right\}$ 
where $n_{\mathrm{g}} \geq 0$ and $n_{\mathrm{d}} \geq 1$. Let $\tau_{\mathrm{D}}\left(n_{\mathrm{g}}, n_{\mathrm{d}}\right)=\mathbb{P}\left\{\mathcal{E}_{i, \mathrm{D}}^{n_{\mathrm{g}}, n_{\mathrm{d}}}\right\}$, then $P_{\mathrm{FA}}$ defined in (11) can be expressed as

$$
\begin{gathered}
P_{\mathrm{D}}=\sum_{n_{\mathrm{g}}=0}^{\infty} \sum_{n_{\mathrm{d}}=1}^{\infty} \tau_{\mathrm{D}}\left(n_{\mathrm{g}}, n_{\mathrm{d}}\right) \mathbb{P}\left\{N_{\mathrm{g}}=n_{\mathrm{g}}, N_{\mathrm{d}}=n_{\mathrm{d}} \mid \theta_{i}=1\right\} \\
=\sum_{n_{\mathrm{g}}=0}^{\infty} \sum_{n_{\mathrm{d}}=1}^{\infty} \tau_{\mathrm{D}}\left(n_{\mathrm{g}}, n_{\mathrm{d}}\right) \frac{\mu_{\mathrm{d}}^{n_{\mathrm{d}}-1} \mu_{\mathrm{g}}^{n_{\mathrm{g}}} \exp \left(-\mu_{\mathrm{d}}-\mu_{\mathrm{g}}\right)}{\left(n_{\mathrm{d}}-1\right) ! n_{\mathrm{g}} !},
\end{gathered}
$$

To find a closed-form expression for $P_{\mathrm{FA}}$ and $P_{\mathrm{D}}$, one has to evaluate the pmf of $\left(\sum_{j \in \mathcal{U}_{i}} Y_{j} \mid N_{\mathrm{g}}, N_{\mathrm{d}}, \theta_{i}\right)$. Consider now the event

$$
\mathcal{Y}_{j, \mathrm{D}}^{n_{\mathrm{g}}, n_{\mathrm{d}}}=\left\{Y_{j}=1 \mid j \in \mathcal{U}_{i}, N_{\mathrm{g}}=n_{\mathrm{g}}, N_{\mathrm{d}}=n_{\mathrm{d}}, \theta_{i}=1\right\}
$$

with $j \in \mathcal{U}_{i}$. For any $j^{\prime} \in \mathcal{U}_{i}$ with $j \neq j^{\prime}, \mathcal{Y}_{j, \mathrm{D}}^{n_{\mathrm{g}}, n_{\mathrm{d}}}$ and $\mathcal{Y}_{j^{\prime}, \mathrm{D}}^{n_{\mathrm{g}}, n_{\mathrm{d}}}$ are dependent. Their dependency comes from the fact that in general $\mathcal{N}_{j} \cap \mathcal{N}_{j^{\prime}} \neq \emptyset$. The pmf of $\sum_{j \in \mathcal{U}_{i}} Y_{j}$ is thus quite difficult to evaluate, since the dependency between the $Y_{j}$ s is not explicit. For this reason, only upper bounds of $P_{\mathrm{FA}}$ and $P_{\mathrm{D}}$ are derived in the following instead of closed analytical forms.

Lemma 5: For any $0<\gamma \leq 1$, one has

$$
\begin{aligned}
& P_{\mathrm{FA}}\left(\mu_{\mathrm{g}}, \mu_{\mathrm{d}}\right)<f_{1}\left(\mu_{\mathrm{g}}, \mu_{\mathrm{d}}\right), \\
& P_{\mathrm{D}}\left(\mu_{\mathrm{g}}, \mu_{\mathrm{d}}\right)<f_{2}\left(\mu_{\mathrm{g}}, \mu_{\mathrm{d}}\right) .
\end{aligned}
$$

\section{Proof: By definition,}

$$
\begin{aligned}
& \tau_{\mathrm{FA}}\left(n_{\mathrm{g}}, n_{\mathrm{d}}\right) \\
& =\mathbb{P}\left\{Y_{i}=1, \frac{\sum_{j \in \mathcal{U}_{i}} Y_{j}}{n_{\mathrm{g}}+n_{\mathrm{d}}}=1 \mid \theta_{i}=0, N_{\mathrm{g}}=n_{\mathrm{g}}, N_{\mathrm{d}}=n_{\mathrm{d}}\right\} \\
& \leq \mathbb{P}\left\{Y_{i}=1 \mid \theta_{i}=0, N_{\mathrm{g}}=n_{\mathrm{g}}, N_{\mathrm{d}}=n_{\mathrm{d}}\right\}=h\left(n_{\mathrm{g}}, n_{\mathrm{d}}\right) .
\end{aligned}
$$

Then from (29), (32), and (36), one obtains (34). Then, (35) can be proved similarly.

\section{B. Proof of Proposition 1}

The following lemma provides sufficient conditions to have monotone left hand-side expressions in (17) and (18).

Lemma 6: Assume that the LODT (3) satisfies Properties 1 and 2, as well as (19) and (20), then, for any $\mu_{10}$,

$$
g_{\mathrm{FA}}\left(\mu_{00}, \mu_{10}\right)=\frac{\mu_{00} P_{\mathrm{FA}}\left(\mu_{00}, \mu_{10}\right)}{\mu_{\mathrm{g}}-\mu_{00}}
$$

is an increasing function of $\mu_{00}$ over $\left[0, \mu_{\mathrm{g}}\right]$ and for any $\mu_{00}$,

$$
g_{\mathrm{D}}\left(\mu_{00}, \mu_{10}\right)=\frac{\mu_{10} P_{\mathrm{D}}\left(\mu_{00}, \mu_{10}\right)}{\mu_{\mathrm{d}}-\mu_{10}}
$$

is an increasing function of $\mu_{10}$ over $\left[0, \mu_{\mathrm{d}}\right]$.

Proof: To prove that $g_{\mathrm{FA}}\left(\mu_{00}, \mu_{10}\right)$ as defined in (37) is monotone increasing in $\mu_{00}$ under the condition (19), one has to show that

$$
\begin{gathered}
\frac{\partial g_{\mathrm{FA}}}{\partial \mu_{00}}=\frac{\mu_{00}}{\mu_{\mathrm{g}}-\mu_{00}} \sum_{n_{\mathrm{g}}=1}^{\infty} \sum_{n_{\mathrm{d}}=0}^{\infty} \frac{\mu_{\mathrm{d}}^{n_{\mathrm{d}}} \mu_{\mathrm{g}}^{n_{\mathrm{g}}-1} \exp \left(-\mu_{\mathrm{d}}-\mu_{\mathrm{g}}\right)}{n_{\mathrm{d}} !\left(n_{\mathrm{g}}-1\right) !} \\
\cdot\left(\tau_{\mathrm{FA}}\left(n_{\mathrm{g}}+1, n_{\mathrm{d}}\right)+\frac{\mu_{00}^{2}-\mu_{00} \mu_{\mathrm{g}}+\mu_{\mathrm{g}}}{\mu_{00}\left(\mu_{\mathrm{g}}-\mu_{00}\right)} \tau_{\mathrm{FA}}\left(n_{\mathrm{g}}, n_{\mathrm{d}}\right)\right)
\end{gathered}
$$

is strictly positive. Therefore, a sufficient condition to have $\frac{\partial g_{\mathrm{FA}}}{\partial \mu_{00}}>0$ is

$$
\tau_{\mathrm{FA}}\left(n_{\mathrm{g}}+1, n_{\mathrm{d}}\right)>-\frac{\mu_{00}^{2}-\mu_{00} \mu_{\mathrm{g}}+\mu_{\mathrm{g}}}{\left(\mu_{\mathrm{g}}-\mu_{00}\right) \mu_{00}} \cdot \tau_{\mathrm{FA}}\left(n_{\mathrm{g}}, n_{\mathrm{d}}\right) .
$$

Since

$$
-\frac{\mu_{00}^{2}-\mu_{00} \mu_{\mathrm{g}}+\mu_{\mathrm{g}}}{\left(\mu_{\mathrm{g}}-\mu_{00}\right) \mu_{00}}=1-\frac{\mu_{\mathrm{g}}}{\left(\mu_{\mathrm{g}}-\mu_{00}\right) \mu_{00}} \leq 1-\frac{4}{\mu_{\mathrm{g}}},
$$

a sufficient condition to have (40) is

$$
\min _{n_{\mathrm{g}} \geq 1, n_{\mathrm{d}} \geq 0} \frac{\tau_{\mathrm{FA}}\left(n_{\mathrm{g}}+1, n_{\mathrm{d}}\right)}{\tau_{\mathrm{FA}}\left(n_{\mathrm{g}}, n_{\mathrm{d}}\right)}>1-\frac{4}{\mu_{\mathrm{g}}} .
$$

One needs to find a lower bound of $\tau_{\mathrm{FA}}\left(n_{\mathrm{g}}+1, n_{\mathrm{d}}\right)$ as a function of $\tau_{\mathrm{FA}}\left(n_{\mathrm{g}}, n_{\mathrm{d}}\right)$. By definition,

$$
\begin{aligned}
& \tau_{\mathrm{FA}}\left(n_{\mathrm{g}}+1, n_{\mathrm{d}}\right) \\
& \stackrel{(a)}{=} \mathbb{P}\left\{Y_{i}=1, \frac{\sum_{j \in \mathcal{B}} Y_{j}}{n_{\mathrm{g}}+n_{\mathrm{d}}+1} \geq \gamma\right\} \\
& \stackrel{(b)}{\geq}\left\{Y_{i}=1, \frac{\sum_{j \in \mathcal{B}} Y_{j}}{n_{\mathrm{g}}+n_{\mathrm{d}}+1} \geq \gamma, \frac{\sum_{j \in \mathcal{B} \backslash\{k\}} Y_{j}}{n_{\mathrm{g}}+n_{\mathrm{d}}} \geq \gamma\right\} \\
& =\mathbb{P}\left\{Y_{i}=1, \frac{\sum_{j \in \mathcal{B} \backslash\{k\}} Y_{j}}{n_{\mathrm{g}}+n_{\mathrm{d}}} \geq \gamma\right\} \\
& \cdot \mathbb{P}\left\{\frac{\sum_{j \in \mathcal{B}} Y_{j}}{n_{\mathrm{g}}+n_{\mathrm{d}}+1} \geq \gamma \mid \frac{\sum_{j \in \mathcal{B} \backslash\{k\}} Y_{j}}{n_{\mathrm{g}}+n_{\mathrm{d}}} \geq \gamma, Y_{i}=1\right\} \\
& \text { (c) } \\
& \stackrel{(c)}{\geq} \tau_{\mathrm{FA}}\left(n_{\mathrm{g}}, n_{\mathrm{d}}\right) \\
& \cdot \mathbb{P}\left\{\frac{\sum_{j \in \mathcal{B}} Y_{j}}{n_{\mathrm{g}}+n_{\mathrm{d}}+1} \geq \gamma \mid \frac{\sum_{j \in \mathcal{B} \backslash\{k\}} Y_{j}}{n_{\mathrm{g}}+n_{\mathrm{d}}} \geq \gamma, Y_{i}=1\right\}
\end{aligned}
$$

where in $(a), \mathcal{B}$ is a set $\mathcal{U}_{i}$ such that $\theta_{i}=0, N_{\mathrm{g}}=n_{\mathrm{g}}+1$, and $N_{\mathrm{d}}=n_{\mathrm{d}}$. In $(b), k$ is an arbitrary node in $\mathcal{N}_{i} \cap \mathcal{G}$. Suppose that an arbitrary network topology is fixed under the condition that $N_{\mathrm{g}}=n_{\mathrm{g}}+1$ and $N_{\mathrm{d}}=n_{\mathrm{d}}$, local tests are performed based on the data vector $\mathbf{M}_{\mathcal{B}}$, and the outcome of local test is $Y_{j}=y_{j}$ for $\forall j \in \mathcal{B}$. Now one removes an arbitrary node $k$ from $\mathcal{N}_{i} \cap \mathcal{G}$ and re-performs the local tests based on the vector $\mathbf{M}_{\mathcal{B} \backslash\{k\}}$, with the outcome $Y_{j}=y_{j}^{\prime}$. Then one is sure that $y_{j} \geq y_{j}^{\prime}$ and

$$
\sum_{j \in \mathcal{B} \backslash\{k\}} y_{j} \geq \sum_{j \in \mathcal{B} \backslash\{k\}} y_{j}^{\prime},
$$

using Property 2. Thus $(c)$ is true. From (43),

$$
\begin{aligned}
& \frac{\tau_{\mathrm{FA}}\left(n_{\mathrm{g}}+1, n_{\mathrm{d}}\right)}{\tau_{\mathrm{FA}}\left(n_{\mathrm{g}}, n_{\mathrm{d}}\right)} \\
& \geq \mathbb{P}\left\{\frac{\sum_{j \in \mathcal{B}} Y_{j}}{n_{\mathrm{g}}+n_{\mathrm{d}}+1} \geq \gamma \mid \frac{\sum_{j \in \mathcal{B} \backslash\{k\}} Y_{j}}{n_{\mathrm{g}}+n_{\mathrm{d}}} \geq \gamma, Y_{i}=1\right\} \\
& \geq \mathbb{P}\left\{Y_{k}=1, \frac{\sum_{j \in \mathcal{B}} Y_{j}}{n_{\mathrm{g}}+n_{\mathrm{d}}+1} \geq \gamma \mid \frac{\sum_{j \in \mathcal{B} \backslash\{k\}} Y_{j}}{n_{\mathrm{g}}+n_{\mathrm{d}}} \geq \gamma, Y_{i}=1\right\}
\end{aligned}
$$




$$
\begin{aligned}
& \geq \mathbb{P}\left\{Y_{k}=1 \mid \frac{\sum_{j \in \mathcal{B} \backslash\{k\}} Y_{j}}{n_{\mathrm{g}}+n_{\mathrm{d}}} \geq \gamma, Y_{i}=1\right\} \\
& \cdot \mathbb{P}\left\{\frac{\sum_{j \in \mathcal{B}} Y_{j}}{n_{\mathrm{g}}+n_{\mathrm{d}}+1} \geq \gamma \mid Y_{k}=1, Y_{i}=1, \frac{\sum_{j \in \mathcal{B} \backslash\{k\}} Y_{j}}{n_{\mathrm{g}}+n_{\mathrm{d}}} \geq \gamma\right\} \\
& \stackrel{(a)}{=} \mathbb{P}\left\{Y_{k}=1 \mid \frac{\sum_{j \in \mathcal{B} \backslash\{k\}} Y_{j}}{n_{\mathrm{g}}+n_{\mathrm{d}}} \geq \gamma, Y_{i}=1\right\} \\
& \stackrel{(b)}{\geq} \mathbb{P}\left\{Y_{k}=1 \mid Y_{i}=1, \theta_{i}=\theta_{k}=0,\right. \\
& \left.N_{\mathrm{g}}=n_{\mathrm{g}}+1, N_{\mathrm{d}}=n_{\mathrm{d}}, k \in \mathcal{N}_{i}\right\},
\end{aligned}
$$

where $(a)$ is by the fact that if $Y_{k}=1$ and $\sum_{j \in \mathcal{B} \backslash\{k\}} Y_{j}>\gamma\left(n_{\mathrm{g}}+n_{\mathrm{d}}\right)$ are true, then one has $\sum_{j \in \mathcal{B}} Y_{j}>\gamma\left(n_{\mathrm{g}}+n_{\mathrm{d}}+1\right)$. Then $(b)$ comes from

$$
\begin{aligned}
\mathbb{P}\left\{Y_{k}=1\right\} & \leq \mathbb{P}\left\{Y_{k}=1 \mid Y_{i}=1\right\} \\
& \leq \mathbb{P}\left\{Y_{k}=1 \mid Y_{i}=1, Y_{j}=1\right\} \leq \cdots
\end{aligned}
$$

see [12] for more details. According to (42) and (45), the first statement of Lemma 6 is proved.

Similarly, a sufficient condition to have $g_{\mathrm{D}}\left(\mu_{00}, \mu_{10}\right)$ an increasing function of $\mu_{10}$ is that

$$
\begin{aligned}
\frac{\tau_{\mathrm{D}}\left(n_{\mathrm{g}}, n_{\mathrm{d}}+1\right)}{\tau_{\mathrm{D}}\left(n_{\mathrm{g}}, n_{\mathrm{d}}\right)} & \geq \mathbb{P}\left\{Y_{k}=1 \mid \theta_{i}=\theta_{k}=1\right\} \\
& \geq q_{\mathrm{D}}(0,2)>1-\frac{4}{\mu_{\mathrm{d}}},
\end{aligned}
$$

which corresponds to the second statement of Lemma 6. Then, we study first the behavior of the right-hand side of (17) for a fixed value of $\mu_{10}$. As shown in Section V-A, $f_{1}$ is a positive and non-decreasing function of $\mu_{00}$, then $1-f_{1}$ is a non-increasing function of $\mu_{00}$. If (19) is satisfied, the left-hand side of (17) is a strictly increasing function of $\mu_{00}$ over $\left[0, \mu_{\mathrm{g}}\right]$. Moreover

$$
g_{\mathrm{FA}}\left(0, \mu_{10}\right)=0<1-f_{1}\left(0, \mu_{10}\right),
$$

and

$$
\lim _{\mu_{00} \rightarrow \mu_{\mathrm{g}}} g_{\mathrm{FA}}\left(\mu_{00}, \mu_{10}\right) \gg 1-\lim _{\mu_{00} \rightarrow \mu_{\mathrm{g}}} f_{1}\left(\mu_{00}, \mu_{10}\right)
$$

since $\lim _{\mu_{00} \rightarrow \mu_{\mathrm{g}}} g_{\mathrm{FA}}\left(\mu_{00}, \mu_{10}\right) \rightarrow \infty$. Therefore, there exists a unique intersection of the graphs of the functions $g_{\mathrm{FA}}\left(\mu_{00}, \mu_{10}\right)$ and $1-f_{1}\left(\mu_{00}, \mu_{10}\right)$ as $\mu_{00}$ goes from 0 to $\mu_{\mathrm{g}}$, for any fixed $\mu_{10}$. The value $\mu_{00}^{+}$of $\mu_{00}$ at which they intersect is thus a function of $\mu_{10}$. One may write $\mu_{00}^{+}=\alpha\left(\mu_{10}\right)$, where $\alpha:\left[0, \mu_{\mathrm{d}}\right] \rightarrow\left[0, \mu_{\mathrm{g}}\right]$. The continuity of $\alpha$ is deduced from that of $f_{1}$ and $g_{\mathrm{FA}}$.

Similarly, one can show that if (20) is satisfied, for any fixed $\mu_{00}$, there exists a unique intersection of the graphs of $g_{\mathrm{D}}\left(\mu_{00}, \mu_{10}\right)$ and $1-f_{2}\left(\mu_{00}, \mu_{10}\right)$ as $\mu_{10}$ varies from 0 to $\mu_{\mathrm{d}}$. The value $\mu_{10}^{+}$of $\mu_{10}$ at which both graphs intersect is thus a function of $\mu_{00}$. One may write $\mu_{10}^{+}=\beta\left(\mu_{00}\right)$, where $\beta$ : $\left[0, \mu_{\mathrm{g}}\right] \rightarrow\left[0, \mu_{\mathrm{d}}\right]$. The continuity of $\beta$ is deduced from that of $f_{2}$ and $g_{\mathrm{D}}$. Then, the equation $\mu_{00}^{*}=\alpha\left(\beta\left(\mu_{00}^{*}\right)\right)$ admits at least a solution from Brouwer's fixed-point theorem, since $\alpha \circ$ $\beta:\left[0, \mu_{\mathrm{g}}\right] \rightarrow\left[0, \mu_{\mathrm{g}}\right]$ is continuous. In this way, Proposition 1 is proved.

\section{ILLUSTRATION}

Assume that each sensor $i$ gets a noisy observation of the same constant scalar physical quantity $\phi$

$$
m_{i}=\phi+w_{i}, \quad \forall i \in \mathcal{S} .
$$

The components $w_{i}$ of the measurement noise in (48) are assumed to be realizations of random variables $W_{i}$. Various outlier models may be considered. Here, we assume that $W_{i} \sim \mathcal{N}\left(E_{i}, \sigma^{2}\right)$, where the bias $E_{i}$ is also a random variable. If $i \in \mathcal{G}$, then $E_{i}$ is uniformly distributed in $[-\Delta, \Delta]$. If $i \in \mathcal{D}$, then $E_{i}$ is uniformly distributed in $[-\xi \Delta,-\Delta] \cup[\Delta, \xi \Delta]$ with $\xi>1$. Moreover, the value of $E_{i}$ of each sensor is constant, i.e., $E_{i}$ does not vary over time.

\section{A. LODT}

Consider some tolerance $\nu>0$ and the interval $\left[m_{i}\right]=$ $\left[m_{i}-\nu, m_{i}+\nu\right]$ of width $2 \nu$ centered around $m_{i}$. Consider now the set estimate [13] of $\phi$ defined as

$$
\left[\widehat{\phi}\left(\mathbf{m}_{\mathcal{A}}\right)\right]=\bigcap_{i \in \mathcal{A}}\left[m_{i}\right] \text {. }
$$
From $\left[\widehat{\phi}\left(\mathbf{m}_{\mathcal{A}}\right)\right]$, one deduces the following low-complexity
LODT

$$
T\left(\mathbf{m}_{\mathcal{A}}\right)= \begin{cases}1, & \text { if }\left[\widehat{\phi}\left(\mathbf{m}_{\mathcal{A}}\right)\right]=\emptyset \\ 0, & \text { else. }\end{cases}
$$

With and without presence of outliers, one is able to evaluate the probability $\mathbb{P}\{[\widehat{\phi}]=\emptyset\}$ as a function of $\sigma$, $\xi$, and $\Delta$. The test (50) involves $\mathbf{m}_{\mathcal{A}}$, but does not need the identity of the node producing the data. Thus $q_{\mathrm{D}}$ and $q_{\text {FA }}$ defined in (4) and (5) only depend on $n_{\mathrm{g}}=|\mathcal{A} \cap \mathcal{G}|$ and $n_{\mathrm{d}}=|\mathcal{A} \cap \mathcal{D}|$. Property 1 is thus satisfied. Assume now that $T\left(\mathbf{m}_{\mathcal{A}}\right)=0$ which implies $\left[\widehat{\phi}\left(\mathbf{m}_{\mathcal{A}}\right)\right] \neq \emptyset$, then by definition of (49), one must have $\left[\widehat{\phi}\left(\mathbf{m}_{\mathcal{A} \backslash\{j\}}\right)\right] \neq \emptyset$ for any $j \in \mathcal{A}$ and Property 2 is satisfied as well.

Now one analyzes the condition in Proposition 1.

Lemma 7: Consider the LODT defined in (49-50), one has

$\mathbb{P}\left\{Y_{k}=1 \mid Y_{i}=1, \theta_{k}=\theta_{i}=0, k \in \mathcal{N}_{i}\right\}>1-\frac{\sqrt{3}}{\pi}-\frac{5}{6 \pi^{2}}$.

The details of the proof may be found in [12].

From (49) and (51), one obtains the constraint of Proposition $1,1-\frac{3 \sqrt{3}}{4 \pi}-\frac{5}{6 \pi^{2}} \geq 1-\frac{4}{\mu_{\mathrm{g}}}$ and thus $\mu_{\mathrm{g}} \leq \frac{24 \pi^{2}}{5+6 \sqrt{3} \pi} \approx 6.3$.

\section{B. Simulation results}

Consider a wireless sensor network (WSN) of 1000 sensors independently and uniformly deployed over a square of size $10 \times 10$ units. To avoid boundary effects, only the sensors in the square of size $\left(10-2 R_{0}\right) \times\left(10-2 R_{0}\right)$ units are considered in the evaluations of $P_{\mathrm{D}}$ and $P_{\mathrm{FA}}$. One sets $\xi=10$ and $\gamma=0.95$. All results have been averaged over 200 independent realizations of the WSN.

The evolution of $P_{\mathrm{D}}$ and $P_{\mathrm{FA}}$ are shown in Figures 1-3 for $\mu \in\{6,20\}, \mu_{\mathrm{d}} / \mu \in\{0.1,0.3\}$ and $\nu / \sigma \in\{1,3,6\}$. The verification of Proposition 2, as wells as the eigenvalues 
TABLE I

Verification of Proposition 2 And the EIgENVAlues $\lambda_{1}$ AND $\lambda_{2}$ OF THE MATRiX A IN (21)

\begin{tabular}{c|c|c|c|c|c|c|c}
$\mu$ & $\frac{\nu}{\sigma}$ & $\frac{\mu_{\mathrm{d}}}{\mu}$ & $Q_{1}$ & $Q_{2}$ & $Q_{3}$ & $\lambda_{1}$ & $\lambda_{2}$ \\
\hline 6 & 6 & 0.1 & -0.12 & 0.96 & 1.01 & 0.12 & -0.10 \\
6 & 3 & 0.1 & -0.02 & 0.89 & 1.07 & 0.19 & -0.10 \\
6 & 1 & 0.1 & 0.02 & 1.56 & 0.48 & -0.38 & -0.50 \\
6 & 6 & 0.3 & -0.10 & 0.84 & 0.97 & 0.34 & -0.28 \\
6 & 3 & 0.3 & -0.10 & 0.93 & 0.98 & 0.35 & -0.28
\end{tabular}
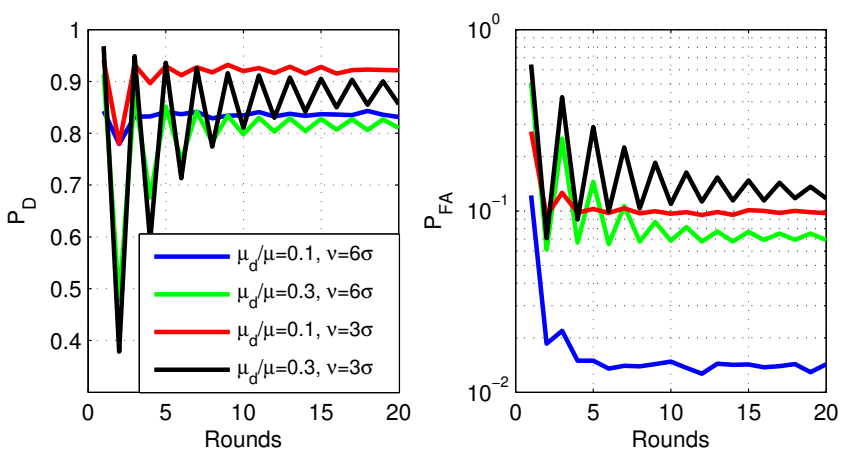

Fig. 1. $\quad P_{\mathrm{D}}$ (left) and $P_{\mathrm{FA}}$ (right) as a function of the number of rounds, with $\mu=6, \mu_{d} / \mu \in\{0.1,0.3\}$, and $\nu / \sigma \in\{3,6\}$.

of the matrix $\mathbf{A}$ in (21), are shown in Table I, with $Q_{1}=$ $A_{11} A_{22}-A_{12} A_{21}, Q_{2}=\left(1-A_{11}\right)\left(1-A_{22}\right)-A_{12} A_{21}$, and $Q_{3}=\left(1+A_{11}\right)\left(1+A_{22}\right)-A_{12} A_{21}$. One sees that the value of $\nu$ and $\mu_{\mathrm{d}} / \mu$ has important influence on the convergence speed of the algorithm: when $\nu$ is large and $\mu_{\mathrm{d}} / \mu$ is small, convergence is faster. The iterative DFD does not converge to a an equilibrium when $\mu=20, \mu_{\mathrm{d}} / \mu=0.1$, and $\nu=\sigma$. Notice that with these values of the parameters, (51) is not satisfied.

\section{CONCLUSIONS AND FUture WORK}

This work proposes a variant of the iterative DFD algorithm proposed in [2] and presents a theoretical analysis of its equilibrium and local asymptotic stability. Sufficient conditions for the existance of a unique and locally stable equilibrium point have been derived. Properties for the uniqueness of the equilibrium are derived independently of the LODT involved at the nodes of the WSN. For a given example, the sufficient conditions translate into upper bounds
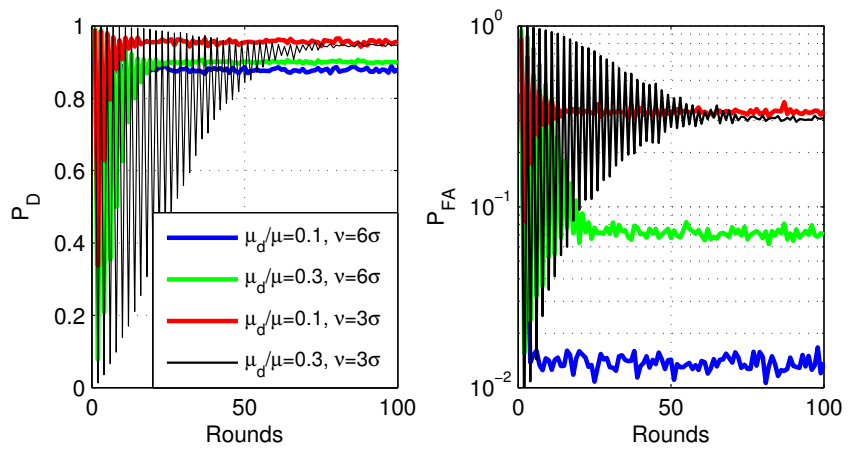

Fig. 2. $\quad P_{\mathrm{D}}$ (left) and $P_{\mathrm{FA}}$ (right) as a function of the number of rounds, with $\mu=20, \mu_{d} / \mu \in\{0.1,0.3\}$, and $\nu / \sigma \in\{3,6\}$.
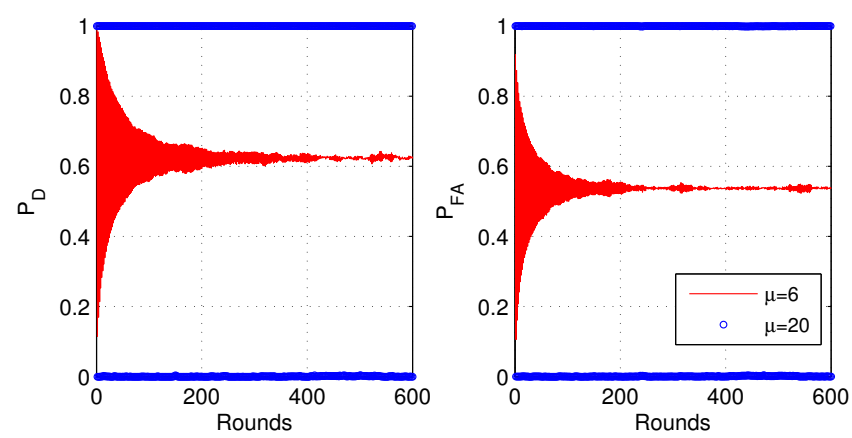

Fig. 3. $\quad P_{\mathrm{D}}$ (left) and $P_{\mathrm{FA}}$ (right) as a function of the number of rounds, with $\mu_{d} / \mu=0.1, \nu / \sigma=1$ and $\mu \in\{6,20\}$.

on $\mu_{\mathrm{g}}$ and $\mu_{\mathrm{d}}$. When these bounds are satisfied, the algorithm behaves well. When they are not satisfied, the equilibrium may not exist.

This iterative algorithm is promising and will be extended to the detection of sparse events.

\section{REFERENCES}

[1] R. R. Wilcox, Introduction to robust estimation and hypothesis testing. Academic Press, 2012.

[2] W. Li, F. Bassi, D. Dardari, M. Kieffer, and G. Pasolini, "Lowcomplexity distributed fault detection for wireless sensor networks," in Proc. IEEE ICC, London, UK, Jun. 2015.

[3] M. Ding, D. Chen, K. Xing, and X. Cheng, "Localized fault-tolerant event boundary detection in sensor networks," in Proc IEEE INFOCOM, Miami, FL, 2005, pp. 902 - 913.

[4] J. Chen, S. Kher, and A. Somani, "Distributed fault detection of wireless sensor networks," in Proc Workshop DIWANS, New York, NY, 2006, pp. $65-72$.

[5] J.-L. Gao, Y.-J. Xu, and X.-W. Li, "Weighted-median based distributed fault detection for wireless sensor networks," Journal of Software, vol. 18, no. 5, pp. 1208 - 1217, 2007.

[6] S. Ji, S.-F. Yuan, T.-H. Ma, and C. Tan, "Distributed fault detection for wireless sensor based on weighted average," in Proc NSWCTC, Wuhan, China, 2010, pp. 57 - 60.

[7] M.-H. Lee and Y.-H. Choi, "Fault detection of wireless sensor networks," Comput. Comm., vol. 31, no. 14, pp. 3469-3475, 2008.

[8] J.-Y. Choi, S.-J. Yim, Y.-J. Huh, and Y.-H. Choi, "A distributed adaptive scheme for detecting faults in wireless sensor networks," WSEAS Trans. on Communications, vol. 8, no. 2, pp. 269-278, 2009

[9] M. Cheraghchi, A. Hormati, A. Karbasi, and M. Vetterli, "Group testing with probabilistic tests: theory, design and application," IEEE Trans. Inf. Theory, vol. 57, no. 10, pp. 7057 - 7067, 2011.

[10] T. Tosic, N. Thomos, and P. Frossard, "Distributed sensor failure detection in sensor networks," Signal Processing, vol. 93, no. 2, pp. 399-410, 2013.

[11] M. Haenggi, M. G. Andrews, F. Baccelli, O. Dousse, and M. F. Franceschetti, "Stochastic geometry and random graphs for the analysis and design of wireless networks," IEEE Journal on Selected Areas in Comm., vol. 27, no. 7, pp. 1029-1046, 2009.

[12] W. Li, F. Bassi, D. Dardari, M. Kieffer, and G. Pasolini, "Lowcomplexity distributed fault detection for wireless sensor networks," in Technical report, available on arxiv.org.

[13] G. Belforte, B. Bona, and V. Cerone, "Parameter estimation algorithms for a set-membership description of uncertainty," Automatica, vol. 26, no. 5, pp. 887-898, 1990. 